\title{
Attributional Style in Healthy Persons: Its Association with 'Theory of Mind' Skills
}

\author{
Im Hong Jeon ${ }^{1,2}$, Kyung Ran Kim,2, Hwan Hee Kim², Jin Young Park ${ }^{1,2}$, Mikyung Lee², \\ Hye Hyun $\mathrm{Jo}^{3}$, Se Jun $\mathrm{Koo}^{2}$, Yu Jin Jeong ${ }^{2}$, Yun Young Song ${ }^{1,2}$, Jee In Kang ${ }^{1,2}$, \\ Su Young Lee ${ }^{2,4}$, Eun Lee ${ }^{1,2}$ and Suk Kyoon $\mathrm{An}^{1,2} \bowtie$ \\ 'Department of Psychiatry, Yonsei University College of Medicine, Seoul, Republic of Korea \\ ${ }^{2}$ Section of Affect and Neuroscience, Institute of Behavioural Science in Medicine, Yonsei University College of Medicine, Seoul, Republic of Korea \\ ${ }^{3}$ Seoul Total Health Care Center, Kangbuk Samsung Hospital, Seoul, Republic of Korea \\ ${ }^{4}$ Department of Psychiatry, Cheil General Hospital \& Women's Healthcare Center, Kwandong University College of Medicine, Seoul, Republic of Korea
}

Objective Attributional style, especially external personal attribution bias, was found to play a pivotal role in clinical and non-clinical paranoia. The study of the relationship of the tendency to infer/perceive hostility and blame with theory of mind skills has significant theoretical importance as it may provide additional information on how persons process social situations. The aim of this study was whether hostility perception bias and blame bias might be associated with theory of mind skills, neurocognition and emotional factors in healthy persons.

Methods Total 263 participants (133 male and 130 female) were recruited. The attributional style was measured by using the Ambiguous Intentions Hostility Questionnaire (AIHQ). Participants were requested to complete a Brüne's Theory of Mind Picture Stories task, neurocognitive task including Standard Progressive Matrices (SPM) and digit span, and other emotional dysregulation trait scales including Rosenberg's self-esteem, Spielberg's trait anxiety inventory, and Novaco anger scale.

Results Multiple regression analysis showed that hostility perception bias score in ambiguous situation were found to be associated with theory of mind questionnaire score and emotional dysregulation traits of Novaco anger scale. Also, composite blame bias score in ambiguous situation were found to be associated with emotional dysregulation traits of Novaco anger scale and Spielberg's trait anxiety scale.

Conclusion The main finding was that the attributional style of hostility perception bias might be primarily contributed by theory of mind skills rather than neurocognitive function such as attention and working memory, and reasoning ability. The interpretations and implications would be discussed in details.

Psychiatry Investig 2013;10:34-40

Key Words Attributional style, Theory of Mind skills, Healthy persons.

\section{INTRODUCTION}

Social cognition is 'the ability to construct representations of the relations between oneself and others, and use those representations flexibly to guide social behavior.' The major domains of social cognition are including the facial emotion perception, social perception, social knowledge, theory of mind and attribution style, which may be separated, but over-

Received: July 11, 2012 Revised: October 22, 2012

Accepted: November 16, 2012 Available online: February 8, 2013

$\triangle$ Correspondence: Suk Kyoon An, MD

Department of Psychiatry, Severance Mental Health Hospital, Yonsei University Health System, 119 Gyeongchung-daero 1926beon-gil, Gwangju 464-100, Republic of Korea

Tel: +82-31-760-9404, Fax: +82-31-761-7582, E-mail: ansk@yuhs.ac

(a) This is an Open Access article distributed under the terms of the Creative Commons Attribution Non-Commercial License (http://creativecommons.org/licenses/by$\mathrm{nc} / 3.0$ ) which permits unrestricted non-commercial use, distribution, and reproduction in any medium, provided the original work is properly cited. lapped. ${ }^{2}$ Among these, attributions are causal statements that infer the word "because". The attribution style has been defined as the pervasive pattern to generate the causal explanation for positive and negative events. Attribution can be divided into internal attribution (i.e., attribution of the cause to oneself) and external attribution (i.e., attribution of the cause to external factors). ${ }^{3}$ External attribution can be further subdivided into external personal attribution (i.e., attributing the cause of event to other people) and external situational attribution (i.e., attributing the cause of event to situational factors). ${ }^{4}$ The biased style of blaming external factors (others or circumstances) rather than oneself when negative events occur (i.e., externalizing bias) has been hypothesized to buffer the self-esteem. ${ }^{5}$ Meanwhile, the bias of external personal attribution rather than external situational attribution was found to play a pivotal role in clinical paranoia, ${ }^{3,4}$ and non-clinical paranoia. ${ }^{6,7}$ 
Theory of mind (ToM) is the ability to represent the mental states of others or to make inferences about other's intentions. ToM skills typically involve the ability to infer intentions, dispositions and beliefs of others. ${ }^{8}$ ToM deficits were found to be an associated factor leading to the persecutory ideation or delusion, ${ }^{9-11}$ although there were inconsistent findings in patients with schizophrenia ${ }^{12,13}$ and in non-clinical participants. ${ }^{14}$ To understand the specific roles of the attributional style and ToM in the formation of paranoia, the interrelations of these two social cognition domains need to be explored. Theoretically, as suggested by Kinderman et al. ${ }^{15}$ for the proper social reasoning of causal explanation (i.e., attribution) of other's behavior, the ability to construct the mental representations of other's mental states (i.e., theory of mind skills) is needed. If someone has difficulty to take other's perspective, he/she would be unable to understand the circumstances that influenced them to behave in a certain way and then, he/she have to be most likely to make external personalizing attributions rather than external situational attributions. In fact, ToM deficit in non-clinical participants were found to be associated with external personal attribution. ${ }^{15,16}$

In addition to the interrelations of attributional bias with ToM, it's association with neurocognition has been also investigated. There are at least two logical reasons to support this association. The first logical one is that to generate situational explanation, as suggested by Langdon et al. ${ }^{17}$ more effortful cognitive search is needed, since blaming others may be easy default. Empirically, the situational information, which shaping other's behavior was found to be not used during the attribution task in healthy participants, if they were in cognitively busy condition. ${ }^{18}$ In chronic schizophrenia and healthy subjects, the some neurocognitive functions were reported to be negatively associated with the externalizing bias. ${ }^{19}$ The second logical reason is the ToM performance may be dependent to the neurocognitive functions such as intelligence, executive function and working memory. ${ }^{15}$ This, as suggested by Kinderman et al. ${ }^{15}$ raised the possibility that the association of attributional style with ToM may be resulted from a third variable such as general neurocognitive or memory ability, although it has not been empirically explored.

Recently, in paranoia study, the attributional style was widely measured by using the Ambiguous Intentions Hostility Questionnaire (AIHQ) ${ }^{20}$ which yielding the hostility perception bias, composite blaming bias, and aggression response in hypothetical negative social scenario in which other's intention is ambiguous, intentional, or accidental. AIHQ may be a suitable measure to understand the interrelationship of the attribution style, ToM, neurocognition and emotional dysregulation factors in respect of paranoia. First of all, it is possible to measure the hostility perception bias that plays an impor- tant role in paranoia process. The tendency to attribute the negative ambiguous situation to other's hostile intention (hostility perception bias) was found to be a significant predictor of paranoia in non-clinical samples ${ }^{20}$ and , in clinical samples. ${ }^{21,22}$ In addition, hostility perception bias in ambiguous situation was repeatedly reported to be correlated with emotional dysregulation traits, such as anxiety and tendency of anger reaction to provocation in non-clinical participants. ${ }^{21,23} \mathrm{Se}$ condly, AIHQ can provide few contextual cues regarding other's intention. In ambiguous situations, which are relatively lacking the situational cues ${ }^{20}$ and thus it may be more cognitively (in both neurocognitive and ToM aspects) demanding to make inference properly on other's behavior. Therefore the socio-cognitive (ToM) and/or neurocognitive deficits and emotional dysregulation factors may be more overtly emerged as significant contributing predictors of the biased attribution style, especially in ambiguous situation.

To our knowledge, there was no study to explore the relationship of attribution style with ToM, neurocognition and emotional dysregulation factors altoghter in the same healthy subjects. The aim of our study was to investigate whether attribution style may be primarily and independently associated with theory of mind skills, general neurocognition such as reasoning and intelligence, and emotional dysreguation traits including tendency of anger reaction to provocation, trait anxiety, self-esteem in healthy persons. Based on the previous reports, ${ }^{15-19}$ our hypothesis was that the biased attributional styles of hostility perception bias and composite blaming bias scores of AIHQ ambiguous situations may be associated with poorer theory of mind skills, lower general neurocognitive functioning and higher emotional dysregulation traits factors in non-clinical samples.

\section{METHODS}

\section{Participants}

Total 263 participants were recruited from an internet job advertisement between January to December in 2011. All participants met the inclusion criteria of being between 15-35 years old. The exclusion criteria were current or past psychiatric or neurological illness history or traumatic brain injury. Written informed consent was obtained from all participants, and the study was reviewed and approved by the Institutional Review Board of Severance Hospital and Severance Mental Health Hospital.

\section{Measures}

Ambiguous Intentions Hostility Questionnaire (AIHQ) The $\mathrm{AIHQ}^{20}$ was an attribution style questionnaire to mea- 
sure the biases of hostility perception, composite blame, and aggressive response. The AIHQ is comprised of 15 hypothetical negative situations. Each situations were varied in intentionality: 5 scenarios were accidental (e.g., "You're dancing at a club and someone bumps into you from behind."), 5 scenarios were ambiguous (e.g., "You walk past a bunch of teenagers at a mall and your hear them start to laugh." ), and 5 scenarios were intentional (e.g., "Your neighbors are playing loud music. You knock on the door and ask them to turn it down. Fifteen minutes later, the music is loud again."). First, participants were asked to imagine the scenario happening to them. And then, they asked to write down what is the reason that other person (or persons) acted that way. The AIHQ yielded hostility perception and aggressive response bias scores and a composite blame bias score. The scales for the hostility perception and aggressive response indices were rated by rater from 1 ("not at all hostile") to 5 ("very hostile"), and 1 ("not at all aggressive") to 5 ("very aggressive"), respectively. The composite blame score (range 1-5.3) is a average score of subjects' ratings of intent (range 1-6; rating about the degree to which the other person committed the act on purpose), anger (range 1-5; rating about how angry the situation would make subject feel), and blame (range 1-5; rating about how much subjects blame the other person for the outcome). The Korean-translated version was reported to have good inter-rater reliability on the hostility perception bias (intra-class correlation: 0.84-0.93) and aggressive response bias (0.71-0.88). ${ }^{24}$ The internal consistency (Cronbach's alpha) of composite blame bias score of AIHQ in the present study, were found to be $0.61-0.68$ across all situations.

The perceived hostility bias, composite blame bias and aggressive response bias scores for ambiguous situations (items $3,5,8,10,13)$ were used in this study according to the proposed analysis strategy of previous studies. ${ }^{20,21}$ To ensure that the ambiguous items were embedded within the measure along with the accidental and intentional situations, all 15 items were administered; the ambiguous items were rated in the context of the accidental and intentional items.

\section{Theory of Mind Picture Stories task}

Theory of Mind Picture Stories task ${ }^{25}$ was developed by Brüne to measure theory of mind ability. Theory of mind was assessed using a novel series of six cartoon picture stories that each consisted of four picture cards. The participants were asked to turn the cards that presented face-down in the same order (4-1-2-3), and to rearrange them in a logical sequence of events. The sequencing score was measured for each picture story. Sequencing score were given two points if the first and last correctly sequenced cards, and one point if the second and third card sequenced correctly (0-36 points). The ques- tionnaire score as consisted of scores of 23 points pertaining to the mental states of the cartoon characters were given (e.g., "What does the blond haired person believe is in the box?", "What does the shopgirl now think the boys intended to do."25

In present study, mean ToM task sequencing score was $33.0( \pm 3.7)$ and mean ToM task questionnaire score was 21.4 $( \pm 2.0)$, which are comparable to the scores of previous ToM picture stories task studies (29.5-36.0, 21.5-29.5, respectibely). ${ }^{26-28}$ In the present study, the internal consistency (Cronbach's alpha) of ToM task sequencing score and questionnaire score were 0.72 and 0.65 in respect.

\section{Neurocognition}

The Standard Progressive Matrices (SPM $)^{29}$ was developed to measure the function of neurocognitive reasoning by analogy and intelligence. It consists of 60 non-coloured diagrammatic puzzles each with a missing part, which the participants were respected to identify from several options. Reliability and validity remain high regardless of whether a timed assessment or not, in various cultural groups. ${ }^{30}$ In present study, among total 263 participants, only 168 participants performed SPM, since SPM was not available to the authors prior to July 2011.

Digit $\operatorname{span}^{31}$ was developed to measure attention and working memory capacity. In the present study, we used computerized version of digit span (http://www.millisecond.com). The digit span is the maximum number of digits that are correctly recalled after listening to a series of digits. The digit span can be assessed for forward recall (=order presented) and for backward recall (=reversed order). In present study, before making two consecutive error in same task, maximum number of digits recalled correctly forward recall was 16 and backward recall were was 15 . In the present study, forward digit span and backward digit span was found to be highly correlated $(\mathrm{r}=0.31, \mathrm{p}<0.001)$, composite score was calculated as mean of two scores. Among total 263 participants, only 171 participants performed digit span task since the computerized version of the digit span task was not available prior to July 2011.

\section{Emotional dysregulation traits}

Novaco Anger Scale (NAS) ${ }^{32}$ was developed to measure once experience of anger. The NAS comprises two main parts, Part A and Part B. Part A was for measure cognitive, arousal, and behavioral domain of anger, and it consists of 48 items. Part B was for measure tendency of anger reaction to provocation, and it consists of 25 items. Part B grouped into five subscales, 1) disrespectful treatment, 2) unfairness/injustice, 3) frustration/interruptions, 4) annoying traits, 5) irritation, by reaction to provocation. The internal consistency (Cronbach's 
alpha) of the NAS was acceptable (Both Part A and B, 0.92) in the previous report. ${ }^{32}$

In the present study, only NAS Part B was used to explore the association between attribution style and tendency of anger reaction to provocation. The internal consistency (Cronbach's alpha) of NAS part B was 0.78 in the present study. Among total 263 participants, only 240 participants performed NAS Part B since Korean version of NAS Part B was not available prior to March 2011.

To measure self-esteem, Rosenberg's self-esteem scale ${ }^{33}$ which is a ten-item self report, was used. Trait anxiety level was assessed using the trait anxiety subscale of Spielberger statetrait anxiety inventory, ${ }^{34}$ a well-known 40 -item instrument which consists of former 20-item for state anxiety and latter 20 -item for trait anxiety. In the present study, only trait anxiety was used to explore the relation of the attribution style with trait anxiety. The internal consistencies (Cronbach's alpha) of Rosenberg's self-esteem scale, and trait anxiety of Spielberger state-trait anxiety inventory in the present study were 0.90 , 0.81 , respectively

\section{Procedures}

Each participant received a pocket of questionnaires, including NAS part B, ${ }^{32}$ Rosenberg's self-esteem scale, ${ }^{33}$ STAI, ${ }^{34}$ and AIHQ. ${ }^{20}$ Each participant performed ToM Picture Stories Task by psychiatrist (JIH) and psychologist (LMK, KSJ), and done $\mathrm{SPM}^{29}$ and computerized digit span task.

\section{Statistical analysis}

In the present study, skewness and kurtosis of all rated scores were in acceptable range $(<1.0)$. The independent $t$-test was done to explore sex difference in AIHQ scores. Preliminarily, Pearson correlations were used to evaluate correlations between AIHQ bias scores in ambiguous situations and other variables. All variables significantly related with AIHQ bias scores were analyzed subsequently using a multiple linear regression to evaluate their independent and primary contributions to AIHQ bias scores. Stepwise method was utilized in the regression model, and pairwise deletion was utilized for missing data. The criterion for significance was set to $\mathrm{p}<0.05$.

\section{RESULTS}

\section{Participant characteristics}

Demographic and clinical characteristics of the subjects were summarized in Table 1. In AIHQ ambiguous situation, there was a significant difference in the composite blame bias scores in ambiguous situations (male 12.3; female 13.5; $\mathrm{p}=$ $0.001)$ by sex.
Table 1. Participant characteristics

\begin{tabular}{lc}
\hline & Participants \\
\hline Age (years) $(\mathrm{N}=263)$ & $21.1(2.9)$ \\
Sex (male/female) $(\mathrm{N}=263)$ & $133 / 130$ \\
Education level (years) $(\mathrm{N}=263)$ & $13.4(1.5)$ \\
Rosenberg's self-esteem35 $(\mathrm{N}=263)$ & $28.0(6.3)$ \\
Trait anxiety of STAI $(\mathrm{N}=263)$ & $41.4(6.7)$ \\
NAS-B $(\mathrm{N}=240)$ & $70.7(12.1)$ \\
Digit span forward $(\mathrm{N}=171)$ & $8.0(0.9)$ \\
Digit span backward $(\mathrm{N}=171)$ & $7.1(1.3)$ \\
SPM (N=168) & $52.0(4.6)$ \\
ToM (N=263) & \\
Sequencing & $33.0(3.7)$ \\
Questionnaire & $21.4(2.0)$ \\
AIHQ Ambiguous situation $(\mathrm{N}=263)$ & \\
Hostility perception bias & $7.8(2.3)$ \\
Blame bias & $12.9(3.1)$ \\
Aggressive response bias & $8.8(1.4)$ \\
\hline
\end{tabular}

STAI: Spielberg's State-Trait Anxiety Inventory, ${ }^{34}$ NAS-B: Novaco Anger Scale ${ }^{32}$ Part B, SPM: Standard Progressive Matrices, ${ }^{29}$ ToM: Theory of Mind Picture Storie Task, ${ }^{25}$ AIHQ: Ambiguous Intentions Hostility Questionnaire ${ }^{20}$

\section{Preliminary correlation analyses of AIHQ score with other variables}

Pearson's correlation analysis revealed that ToM task questionnaire score was found to be correlated with hostility perception bias scores in ambiguous situations. There was no any other correlation between attribution style scores and neurocognitive measures of SPM and digit span composite score. The NAS Part B score was shown to be correlated with hostility perception bias and composite blame bias scores and trait anxiety was correlated with composite blame bias in ambiguous situation, while Rosenberg's self-esteem was not related to any bias scores. There were also significant correlations of age and education level with some attribution styles. Summary of these correlations can be found in Table 2 .

\section{Multiple linear regression analysis of AIHQ score with other variables}

Overall full regression model was significant and the predictors accounted for $7.4 \%$ of the variance in hostility perception bias and for $18.9 \%$ of the variance in composite blame bias in ambiguous situations. In the regression model of hostility perception bias, ToM task questionnaire score, emotional dysregulation trait factors, especially NAS-B, and age were emerged as primary predicting factors. In the regression model of composite blame bias, there were also independent predictors including emotional dysregulation traits such as NAS$B$, trait anxiety and sex (female) for ambiguous situations. There was no independent associated factor of aggressive re- 
Table 2. Preliminary correlation analyses of AIHQ score with other variables

\begin{tabular}{lccc}
\hline & & AIHQ (Ambiguous) \\
\cline { 2 - 3 } & Hostility perception bias & Blame bias & Aggressive response bias \\
\hline Age (years) (N=263) & $-0.19^{* *}$ & $-0.13^{*}$ & -0.05 \\
Education level (years) (N=263) & $-0.17^{* *}$ & -0.01 & 0.04 \\
Rosenberg's self-esteem (N=263) & -0.08 & -0.08 & 0.06 \\
Trait anxiety of STAI (N=263) & 0.07 & $0.23^{*}$ & 0.04 \\
NAS-B (N=240) & $0.18^{* *}$ & $0.37^{* *}$ & 0.07 \\
Digit span composite score (N=171) & -0.05 & -0.10 & 0.02 \\
SPM (N=168) & -0.10 & 0.07 & 0.09 \\
ToM sequencing (N=263) & -0.05 & 0.01 & 0.01 \\
Questionnaire (N=263) & $-0.15^{*}$ & 0.06 & -0.08 \\
\hline
\end{tabular}

${ }^{*} \mathrm{p}<0.05,{ }^{* *} \mathrm{p}<0.01$. STAI: Spielberg's State-Trait Anxiety Inventory, ${ }^{34}$ NAS-B: Novaco Anger Scale ${ }^{32}$ Part B, SPM: Standard Progressive Matrices, ${ }^{29}$ ToM: Theory of Mind Picture Storie Task, ${ }^{25}$ AIHQ: Ambiguous Intentions Hostility Questionnaire ${ }^{20}$

Table 3. Multiple linear regression analysis of AIHQ score with other variables

\begin{tabular}{|c|c|c|c|c|c|c|c|c|c|}
\hline \multirow{2}{*}{ AIHQ (Ambiguous) } & \multirow{2}{*}{ Determinants } & \multirow{2}{*}{$\beta^{*}$} & \multicolumn{4}{|c|}{ Participants } & \multirow{2}{*}{$\mathrm{F}$} & \multirow{2}{*}{ df } & \multirow{2}{*}{$\mathrm{p}$} \\
\hline & & & $\mathrm{t}$ & $\mathrm{p}$ & $\Delta \mathrm{R}^{2 \dagger}$ & $\mathrm{R}^{2 \ddagger}$ & & & \\
\hline \multirow[t]{3}{*}{ Hostility perception bias } & Age & -0.17 & -2.77 & 0.006 & 0.035 & 0.074 & 7.3 & 3,236 & $<0.001$ \\
\hline & NAS-B & 0.18 & 2.84 & 0.005 & 0.029 & & & & \\
\hline & ToM-Q & -0.15 & -2.36 & 0.019 & 0.022 & & & & \\
\hline \multirow[t]{3}{*}{ Blame bias } & NAS-B & 0.34 & 5.85 & $<0.001$ & 0.139 & 0.189 & 19.6 & 3,236 & $<0.001$ \\
\hline & Trait anxiety of STAI & 0.17 & 2.96 & 0.003 & 0.035 & & & & \\
\hline & Sex (female) & 0.16 & 2.76 & 0.006 & 0.026 & & & & \\
\hline
\end{tabular}

${ }^{*}$ standardized coefficient, ${ }^{\dagger}$ changed variance, $\Delta \mathrm{R}^{2}$, ${ }^{\ddagger}$ adjusted $\mathrm{R}^{2}$, Explained variance by model. STAI: Spielberg's State-Trait Anxiety Inventory, ${ }^{34}$ NAS-B: Novaco Anger Scale ${ }^{32}$ Part B, SPM: Standard Progressive Matrices, ${ }^{29}$ ToM: Theory of Mind Picture Storie Task, ${ }^{25}$ AIHQ: Ambiguous Intentions Hostility Questionnaire, ${ }^{20}$ VIF: variance inflation factor

sponse bias. The tolerance among the predictors did not indicate multi-collinearity (all VIF $<1.1$ ). A summary of regression analysis was shown in Table 3.

\section{DISCUSSION}

To our knowledge this is the first study to investigate the associations of attributional style such as hostility perception bias and composite blame bias scores in ambiguous situations of AIHQ with the socio-cognition, neurocognition, and emotional dysregulation traits factors altogether in the same healthy people. The main findings of this study were that hostility perception bias score was primarily and independently associated with ToM picture stories task questionnaire score, tendency of anger reaction to provocation indexed by NAS Part B and age in ambiguous situations, and composite blame bias score were independently associated with tendency of anger reaction to provocation indexed by NAS Part B, trait anxiety and sex.

In regard of theory of mind skills on the attribution style, the hostility perception bias was found to be explained by ToM questionnaire score in hypothetical negative situations in which other's intention is ambiguous. This finding is grossly compatible to the previous findings that external personalizing bias of IPSAQ ${ }^{6}$ and ToM skills were inversely associated. ${ }^{15-17}$ The constructs of hostility perception bias of AIHQ and external personalizing attribution bias in negative situations of IPSAQ may be very similar and overlapped, which was supported by the observed strong correlation $(r>0.30)$ of these two variables in the previous clinical paranoia study. ${ }^{21}$ For correct perception of the other's hostility, it should be needed to take other's perspective and then to understand the circumstances which shaping other's behavior in negative situations in which other's intention is ambiguous. If someone has poorer ToM skills, he/she has some difficulties of the perspective taking and do not consider the situational factors influencing other's behavior, which in turn, may result in biased hostility perception of other's intention. Thus, if someone has deficit in theory of mind skills, he/she might interpret world as more threatening and hostile, as a result he/she could be paranoid. Meanwhile, composite blame bias was not found to be associated with any ToM ability. The possible explanation was that the two attribution bias index of hostility perception and composite blame bias score may reflect the somewhat different aspects of attribution style. In fact, some previous studies ${ }^{15,35}$ found that comparing self (composite blame score) 
versus independent rater's rating (hostility perception score) scores leads to different findings. In addition, composite blame score, which is composed of the intent, anger and blame aspects, may reflect more affective component, while hostility perception bias may reflect more perceptual-cognitive component of the attribution bias. Thus, ToM Picture Stories questionnaire score, which is cognitive rather emotional, may be likely to be associated with the hostility perception bias than composite blame bias. Another possible explanation is that it might be possible that to the other's mischievous and playful act, someone may feel it as purposeful, be provoked to be angry, and thus blame other, while he/she do not perceive this act as an intentional behavior to harm to him/herself. If his/ her ToM ability is within normal range, he/she may rate the composite blame score as high or not according to his/her past experience, while his/her expressions on the hostility perception are not to be rated as high. If his/her ToM ability is compromised, he/she may express the both attribution scores as high. Thus, ToM ability might be primarily and independently associated with the attribution tendency to perceive other's behavior as more intentionally threatening and hostile (hostility perception) but not with tendency of blaming others (composite blame score).

Regarding the neurocognition on attribution style, contrary to our hypothesis, which was based on the above-mentioned logical reasons ${ }^{15,17}$ and empirical findings in chronic schizophrenia patients, ${ }^{18,19}$ there was no significant primary association of the neurocognitive function of digit span and SPM with any attribution biased style. The possible explanation is that attention and working memory indexed by composite digit span score, and reasoning ability and intelligence indexed by SPM may be not associated with the biased attribution style even in ambiguous situation at least in healthy subjects who is not under the cognitive busy condition. For clarification, it would be needed to construct the attribution task with cognitively busy condition just like Gilbert et al.s study ${ }^{18}$ or to conduct the research in the cognitively impaired clinical subjects such as schizophrenia.

For emotional dysregulation trait factors, the tendency of anger reaction to provocation indexed by NAS Part B were found to be a significant predictor of attribution style such as hostility perception bias and composite blame bias in ambiguous situations. This is a compatible finding with previous reports of significant relations of aggression questionnaire with the broad attribution bias of AIHQ in nonclinical samples. ${ }^{21,23}$ The trait anxiety score was found to be associated with composite blame bias not with hostility perception bias. This finding may explained by that the trait anxiety may be more likely to be associated with the more affective attribution bias of composite blame score than with the more perceptuo-cogni- tive attribution bias of hostility perception score (see above discussion).

In addition, age and sex was significantly associated with some attribution style of AIHQ. This finding means that these demographic factors should be considered as independent contributing factors in the attribution style research.

It is noteworthy that the ToM, though significant, contributed only small (about 2\%) explanation of the variance in attribution style in the present study. Even after additions of other emotional traits factors and demographic variables, the variances of attribution styles were only modestly explained (for hostility perception bias, 7.4\%; for composite blame bias, $18.9 \%)$. However, a previous report ${ }^{19}$ also reported that the comprehensive cognitive function contributed somewhat modestly to variance in attribution style in healthy subjects (9.7\%) and as well as schizophrenia patients (10.5-21.2\%). This relatively small-to-moderate sized explanation of variance may be derived partly from the complexity of the attribution style.

This study had a several limitations. One limitation was that the present findings could not be generalized to clinical samples, since all participants were healthy subjects. In near future, it should be explored these associations to be emerged also in large clinical subjects. Another limitation was that the data of the SPM and computerized digit span were not available in some subjects. Thus, the main findings of the present study should be cautiously interpreted. Lastly, the cognitive measures were not comprehensive one. In near future, the comprehensive neurocognitive functional assessments should be conducted and explored the relationship of the neurocognitive function with the attribution style.

In summary, the present findings suggest that the hostility perception of the attributional style, in hypothetical negative social context in which other's intention is ambiguous, may be primarily and independently contributed by the theory of mind skills rather than neurocognitive function of attention and working memory and reasoning ability. In future study, it would be valuable to explore whether these primary relationships of attribution style with theory of mind skills might be also observed in clinical populations such as people with schizophrenia or at ultra-high risk for psychosis who are consistently showing the paranoid symptoms.

\section{Acknowledgments}

This study was supported by the National Research Foundation of Korea (NRF) grant funded by the Korea Government (MEST)(No. 2010-002 4264).

\section{REFERENCES}

1. Adolphs R. The neurobiology of social cognition. Curr Opin Neurobiol 2001;11:231-239.

2. Kerr RS, Horan WP. Definition and Measurement of Neurocognition 
and Socialcognition. In: Roder V, Medalla A, Editors. Neurocognition and Social Cognition in Schizophrenia Patients. Basic Concepts and Treatment. Key Issues Mental Health. Basel: Karger, 2010, p.1-22.

3. Kinderman P, Bentall RP. Causal attributions in paranoia and depression: internal, personal, and situational attributions for negative events. J Abnorm Psychol 1997;106:341-345.

4. Kinderman P, Bentall RP. Self-discrepancies and persecutory delusions: evidence for a model of paranoid ideation. J Abnorm Psychol 1996;105:106-113.

5. Mezulis AH, Abramson LY, Hyde JS, Hankin BL. Is there a universal positivity bias in attributions? A meta-analytic review of individual, developmental, and cultural differences in the self-serving attributional bias. Psychol Bull 2004;130:711-747.

6. Kinderman P, Bentall RP. A new measure of causal locus: the internal, personal and situational attribution questionnaire. Pers Indiv Dif 1996; 20:261-264.

7. McKay R, Langdon R, Coltheart M. Paranoia, persecutory delusions and attributional biases. Psychiatry Res 2005;136:233-245.

8. Frith CD, Frith U. The neural basis of mentalizing. Neuron 2006;50: 531-534.

9. Frith CD. The Cognitive Neuropsychology of Schizophrenia. Hove, UK: Psychology Press: 1992

10. Corcoran R, Mercer G, Frith CD. Schizophrenia, symptomatology and social inference: investigating "theory of mind" in people with schizophrenia. Schizophr Res 1995;17:5-13.

11. Frith CD, Corcoran R. Exploring 'theory of mind' in people with schizophrenia. Psychol Med 1996;26:521-530.

12. Drury VM, Robinson EJ, Birchwood M. ‘Theory of mind' skills during an acute episode of psychosis and following recovery. Psychol Med 1998; 28:1101-1112.

13. Greig TC, Bryson GJ, Bell MD. Theory of mind performance in schizophrenia: diagnostic, symptom, and neuropsychological correlates. J Nerv Ment Dis 2004;192:12-18.

14. Fernyhough C, Jones SR, Whittle C, Waterhouse J, Bentall RP. Theory of mind, schizotypy, and persecutory ideation in young adults. Cogn Neuropsychiatry 2008;13:233-249.

15. Kinderman P, Dunbar R, Bentall RP. Theory of mind deficits and causal attributions. Br J Psychol 1998;89:191-204.

16. Taylor JL, Kinderman P. An analogue study of attributional complexity, theory of mind deficits and paranoia. Br J Psychol 2002;93:137-140.

17. Langdon R, Corner T, McLaren J, Ward PB, Coltheart M. Externalizing and personalizing biases in persecutory delusions: the relationship with poor insight and theory-of-mind. Behav Res Ther 2006;44:699-713.

18. Gilbert DT, Pelham BW, Krull DS. On cognitive busyness when person perceivers meet persons perceived. J Pers Soc Psychol 1988;54:733740 .

19. Donohoe G, Spoletini I, McGlade N, Behan C, Hayden J, O’Donoghue
T, et al. Are relational style and neuropsychological performance predictors of social attributions in chronic schizophrenia? Psychiatry Res 2008;161:19-27.

20. Combs DR, Penn DL, Wicher M, Waldheter E. The Ambiguous Intentions Hostility Questionnaire (AIHQ): a new measure for evaluating hostile social-cognitive biases in paranoia. Cogn Neuropsychiatry 2007; 12:128-143.

21. Combs DR, Penn DL, Michael CO, Basso MR, Wiedeman R, Siebenmorgan M, et al. Perceptions of hostility by persons with and without persecutory delusions. Cogn Neuropsychiatry 2009;14:30-52.

22. An SK, Kang JI, Park JY, Kim KR, Lee SY, Lee E. Attribution bias in ultra-high risk for psychosis and first-episode schizophrenia. Schizophr Res 2010;118:54-61.

23. Combs DR, Penn DL, Cassisi J, Michael C, Wood T, Wanner J, et al. Perceived racism as a predictor of paranoia among African Americans. J Black Psychol 2006;32:87.

24. Chang H, Lee SK, Kim KR, Lee SY, Park JY, Kim EJ, et al. Development of Korean Version of the Ambiguous Intentions Hostility Questionnaire (K-AIHQ). J Korean Neuropsychiatr Assoc 2009;48:29-35.

25. Brüne M. Social Cognition and Behaviour in Schizophrenia. In: Brqne M, Ribbert H, Schiefenhövel W, Editors. The Social Brain: Evolution and Pathology. Chichester: John Wiley, 2003, p.277-313.

26. Brune M. Emotion recognition, 'theory of mind,' and social behavior in schizophrenia. Psychiatry Res 2005;133:135-147.

27. Bommer I, Brune M. Social cognition in "pure" delusional disorder. Cogn Neuropsychiatry 2006;11:493-503.

28. Brune M, Lissek S, Fuchs N, Witthaus H, Peters S, Nicolas V, et al. An fMRI study of theory of mind in schizophrenic patients with "passivity" symptoms. Neuropsychologia 2008;46:1992-2001.

29. Raven J, Raven JC, Court JH. Manual for Raven's Progressive Matrices and Vocabulary Scales (1998 Edition). Oxford: Oxford Psychologists Press; 1998.

30. Raven J. The Raven's progressive matrices: change and stability over culture and time. Cogn Psychol 2000;41:1-48.

31. Lumiley FH, Calhoon SH. Memory span for words presented auditorially. Appl Psychol 1934;18:773-784.

32. Novaco RW. Anger as a Risk Factor for Violence among the Mentally Disordered. In: Monahan J \& Steadman HJ, Editors. Violence and Mental Disorder: Developments in Risk Assessment. Chicago: University of Chicago Press; 1994.

33. Rosenberg M. Society and the Adolescent Self-Image. Revised Edition. Middletown, CT: Wesleyan University Press; 1989

34. Spielberger CD. Manual for the State-Trait Anxiety Inventory: STAI (Form Y). Palo Alto, CA: Consulting Psychologists Press; 1983.

35. Randall F, Corcoran R, Day JC, Bentall RP. Attention, theory of mind, and causal attributions in people with persecutory delusions: A preliminary investigation. Cogn Neuropsychiatry 2003;8:287-294. 